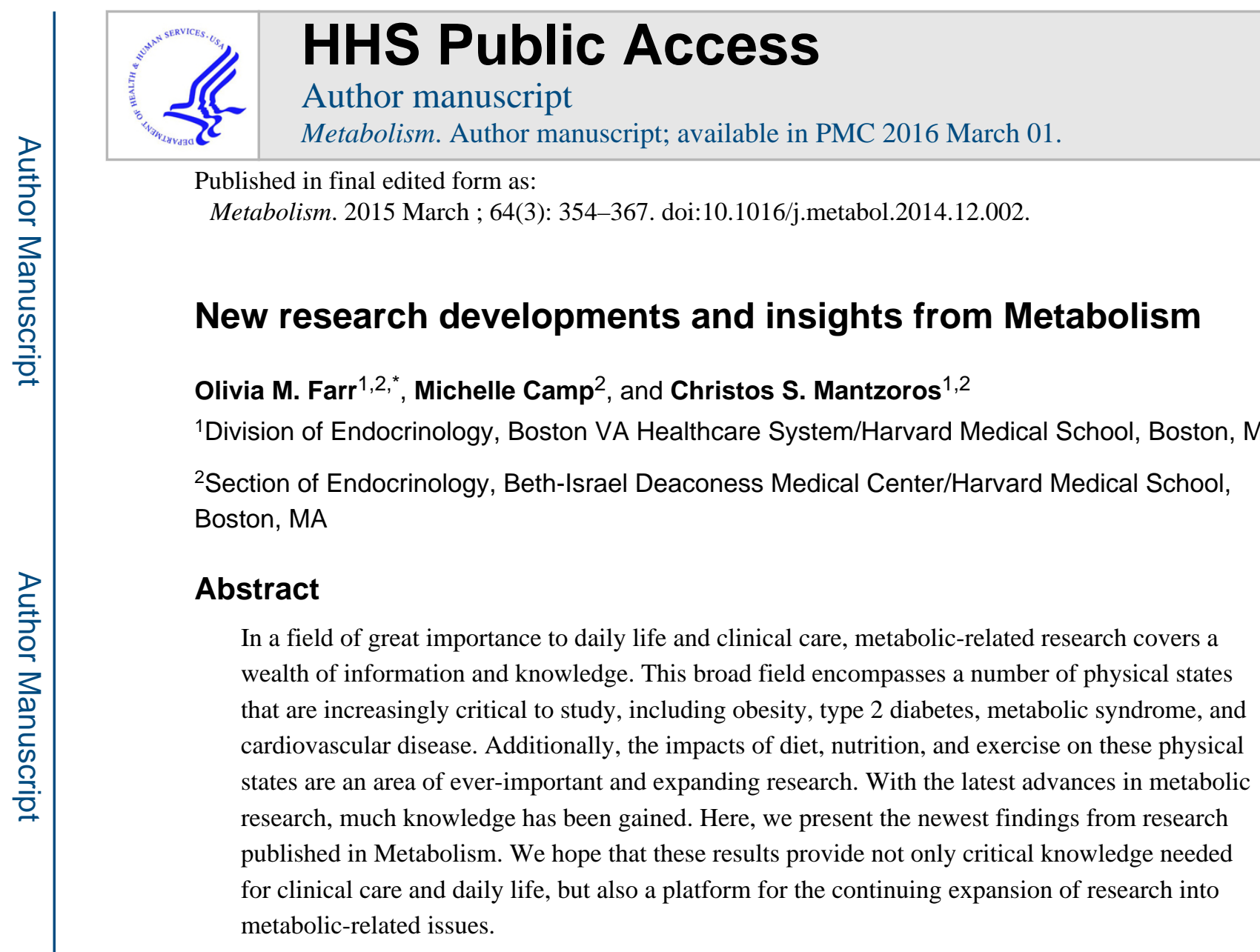

\title{
Diet and Nutrition
}

\section{Carbohydrate influences on body composition in polycystic ovary syndrome}

In order to target healthy weight loss, diet composition may require special consideration for women with poly-cystic ovary syndrome. Goss et al. (1) demonstrated in a crossover study of 30 women with poly-cystic ovary syndrome (aged $31 \pm 5.8$ years) that consumption of a reduced-carbohydrate diet as compared to a standard diet significantly decreased the amount of adipose tissue without changing total calories consumed over the course of eight weeks. While on the low carbohydrate diet, loss of fat mass occurred from subcutaneousabdominal, intra-abdominal, and thigh-intermuscular adipose tissues $(-4.6 \%,-7.1 \%$, and $-11.5 \%$, respectively). Furthermore, the reduced-carbohydrate diets were also associated with decreased insulin levels. In contrast, the "standard" diet may have decreased lean mass by converting it to fat. Therefore, women with polycystic ovary syndrome who consume a diet lower in carbohydrates may preferentially lose fat mass from unhealthy areas of the

\footnotetext{
(C) 2014 Published by Elsevier Inc.

*Address correspondence to: Olivia Farr, VA Boston Healthcare System, Division of Endocrinology (9-B), 150 S. Huntington Ave., Boston, MA 02130, Phone: 857-364-4233, Olivia.Farr@ va.gov.

Disclosure Statement: The authors have nothing to disclose.

Publisher's Disclaimer: This is a PDF file of an unedited manuscript that has been accepted for publication. As a service to our customers we are providing this early version of the manuscript. The manuscript will undergo copyediting, typesetting, and review of the resulting proof before it is published in its final citable form. Please note that during the production process errors may be discovered which could affect the content, and all legal disclaimers that apply to the journal pertain.
} 
body. Future studies could be focused on whether altering fat or protein content has a similar effect on the loss of fat mass in women with polycystic ovary syndrome.

\section{Impacts of ginsenosides on hyperlipidemia and GLP-1}

Ginsenosides, found in Panax Ginseng, help to ameliorate hyperlipidemia, but the mechanism by which they act is still not yet completely understood. Liu et al. (2) investigated whether glucagon-like peptide-1 (GLP-1) release mediated by ginseng total saponins (GTS), in addition to exerting anti-diabetic properties, have effects on hyperlipidemia in 20 obese male Sprague-Dawley rats (weighing $100 \mathrm{~g}-200 \mathrm{~g}$ ). After the rats were randomized to receive either a high-fat diet (HFD) intervention or a chow control diet for four weeks, rats on the HFD were further randomized to a treatment of low-dose (150 $\mathrm{mg} / \mathrm{kg} /$ day) or high-dose (300 mg/kg/day) GTS for an additional four weeks. Liver weight in rats fed a HFD decreased by $6.8 \%$ and $7.8 \%$ after the low- and high-dose treatments, respectively. As measures of body fat content, epididymal fat and retroperitoneal fat decreased $21 \%$ and $16 \%$, respectively, in rats treated with high-dose GTS as compared to HFD control rats. Similarly, plasma levels of triglycerides, total cholesterol, and free fatty acids decreased by 39\%, 15\% and 16\%, respectively, with high-dose treatment. Lastly, plasma levels of Apo-B48 and LDL-C decreased by about $38 \%$ and $28 \%$, respectively, again with high-dose GTS treatment. Moreover, treatment with ginsenosides improved insulin resistance and leptin sensitivity and increased GLP-1 levels. Although it was determined that oral ginsenosides may mediate the anti-hyperlipidemic effects through greater GLP-1 secretion, future research should consider whether oral ginsenosides may have a direct effect on lowering lipid profiles.

\section{Vitamin D influences diabetic outcomes}

Vitamin D deficiency often accompanies type 2 diabetes, but the potential role of vitamin D in the pathogenesis of diabetes, if any, remains unclear. Kampmann et al. (3) sought to determine whether administration of vitamin $\mathrm{D}$ in 16 subjects with type 2 diabetes who had a vitamin D deficiency would positively affect insulin and inflammatory markers. In a randomized double-blind trial with 16 participants, 8 adults (aged $61.6 \pm 4.4$ years) received oral cholecalciferol ( $280 \mu \mathrm{g} /$ day for two weeks and $140 \mu \mathrm{g} /$ day the following 10 weeks) and 8 additional adults (aged $57 \pm 4.5$ years) were given placebo. Plasma 25 -hydroxyvitamin D (25OHD) significantly increased $238 \%(\mathrm{p}=0.01)$ in the supplemented group, whereas $25 \mathrm{OHD}$ decreased $7.8 \%$ in the placebo group $(\mathrm{p}=0.02)$. Serum- $1,25(\mathrm{OH})_{2}$ also increased by about $40 \%$ in the treatment group. C-peptide levels, incremental AUC insulin and insulin secretory burst mass showed a trend towards improvement, which, however, did not reach the level of statistical significance ( $p=0.05-0.10)$, indicating a potential improvement in insulin secretion. Insulin sensitivity and inflammation, however, did not improve with vitamin D replacement therapy. Future research with larger sample sizes and longer treatment duration could help elucidate further benefits of vitamin D supplements for type 2 diabetes.

\section{Fish-based diets and endothelial function}

To test whether a fish-based diet with high $\mathrm{n}-3$ polyunsaturated fatty acids ( $>3.0 \mathrm{~g} /$ day) may improve endothelial function as compared to a control diet, Kondo et al. (4) enrolled 23 
postmenopausal women with type 2 diabetes (aged $69.7 \pm 6.6$ years) in a randomized, crossover trial to consume either the fish-based or control diet for 4 weeks followed by the opposite for the next four weeks. Determinants of endothelial function improved with the fish-based diet: peak forearm blood flow by $63.7 \%$, duration of reactive hyperemia by $27.9 \%$ and flow debt repayment by $70.7 \%$. While the fish-based diet as compared to the control diet resulted in improvements in endothelial function, these observations did not correlate with n-3 polyunsaturated fatty acid levels, suggesting that something else in the fish diet may be causing these improvements. Future studies should examine this in more general populations, as well as more broadly seek to discover the beneficial component in the fish-based diet.

\section{High protein diets influences on hormones}

Henning et al. (5) sought to determine whether a diet high in protein during an energy deficit may attenuate the typically observed fat-free mass loss through insulin-like growth factor 1 (IGF-1) signaling. Using a block-randomized design, 33 adult participants were assigned to a diet with protein levels of either the recommended daily allowance (RDA) (aged: $22 \pm 1$ years), twice the RDA (aged: $21 \pm 1$ years) or three times the RDA (aged: $22 \pm 1$ years; 0.8 , 1.6 , or $2.4 \mathrm{~g} / \mathrm{kg} /$ day, respectively) for 31 days. Hormone levels were measured and compared after 10 days of a weight-maintaining diet and 21 days later after a $40 \%$ energydeficient diet. Energy deficit decreased IGF-1 levels (14\%) and testosterone levels (total: 16\%, free: $23 \%$ ) and increased IGF binding proteins (63\%), regardless of protein level intake. Changes in these molecules did not correlate with changes in fat-free mass seen after energy deficit, suggesting that they may not play a role in mediating the relationships between energy-deficient diets and fat-free mass regardless of high protein intake. Future studies should examine other potential mechanisms to prevent loss of fat-free mass during energy deficient diets.

\section{Influences of maternal diet on insulin resistance in the offspring}

To determine whether a mother's HFD during pregnancy or lactation would affect insulin resistance in their offspring, Melo et al. (6) examined newborn or recently weaned mice (6 animals per litter) from ten mothers who were randomly assigned to receive a HFD or control diet. Recently weaned ( 28 days old), but not newborn mice ( 0 days old), showed elevated markers for endoplasmic reticulum stress and triglycerides. Thus, these results suggest that maternal diet may have more influence over offspring metabolic dysfunction during lactation rather than pregnancy. Future studies should examine how this may continue into adulthood risk of obesity and metabolic comorbidities.

\section{Balanced high fat diets improve cardiometabolic risk}

Silver et al. (7) hypothesized that a balanced high fat diet (9g/day) for 16 weeks, containing equal amounts of saturated, monounsaturated, and polyunsaturated fats, would lessen cardiovascular disease risk and inflammation. The 144 obese, premenopausal women (aged $36.7 \pm 6.8$ years) stabilized on the HFD for 2 weeks were subsequently randomized to supplement their diet with either encapsulated stearate (18:0), oleate(18:1), linoleate (18:2) or placebo for 12 weeks. Body fat mass decreased by about $2.5 \pm 2.1 \%$ while lean mass increased by about $2.5 \pm 2.1 \%$. Additionally, multiple inflammatory marker levels decreased 
in all groups. HFD supplemented with stearate most effectively reduced the inflammatory marker interferon gamma by $74 \%$, while HFD supplemented with linoleate most markedly decreased plasminogen activator inhibitor-1, a measure of vascular function, by $31 \%$. These results may suggest that balancing fats would improve cardiovascular disease risk in women. However, future studies should expand these results by testing the effects on broader populations, including men and postmenopausal women, and studying any potential longterm effects

\section{Walnuts may help improve metabolic risk factors}

In a controlled crossover study comparing a walnut-enriched diet to a control diet, Wu et al. (8) examined whether walnuts may improve biomarkers for metabolic risk, including lipids, inflammatory markers, and adipokines. Forty participants (aged $60 \pm 1$ years) were randomized to consume either a walnut-enriched diet ( $43 \mathrm{~g} /$ day) followed by a control diet or the reverse order, each for a period of 8 weeks. Consumption of a walnut-rich diet, placebo-adjusted, resulted in a $4.7 \%$ decrease in non-HDL cholesterol levels and a $5.4 \%$ decrease in apolipoprotein-B levels, findings that may implicate how walnuts help to reduce cardiovascular disease risk. Other markers of metabolic dysfunction such as insulin resistance, inflammatory markers, and adipokines were not affected. Future research should further define the relationship between walnut consumption and cardiovascular risk as well as determine the long-term impacts of a walnut-enriched diet on heart health.

\section{Coffee and green tea consumption on diabetes risk}

To determine if coffee or green tea may influence biomarkers of diabetes, Pham et al. (9) collected data through cross-sectional epidemiological surveys from 1440 Japanese participants aged 18-69 years. In overweight participants only, higher coffee consumption was associated with a significant decrease in insulin resistance as measured by homeostatic model assessment of insulin resistance (HOMA-IR; $p$ for trend $=0.03$ ), while green tea consumption surprisingly was associated with an increase in HOMA-IR ( $p$ for trend $=0.02$ ). Neither coffee nor green tea consumption was associated with glycated hemoglobin (HbA1c) levels. This study may suggest other benefits of coffee with regards to diabetes risk, though this would need to be confirmed in future, placebo-controlled studies. Additionally, the difference in caffeine between coffee and green tea may have influenced the results and future research should determine if caffeine is a potential factor contributing to associations with insulin resistance.

\section{Western vs. Mediterranean diets' influence on inflammatory markers}

Whether and how healthy or unhealthy Western or Mediterranean diet patterns may influence inflammatory biomarkers and adipokines is not yet clear. Therefore, Park et al. (10) examined in 151 participants (aged > 37 years) whether these markers may be associated with healthy Western and Mediterranean diets through food frequency questionnaires and cross-sectional associations among dietary parameters. C-reactive protein, an inflammatory marker, was negatively correlated ( $<<0.001)$ with "healthy" versions of both diets even when possible confounders were controlled, suggesting increased inflammation with poorer diets. Irisin was not associated with healthy versions of these diets, although it was correlated with body mass index (BMI; $\mathrm{p}=0.008)$, waist circumference 
$(\mathrm{p}=0.003)$, and fat mass $(\mathrm{p}=0.01)$, which often result from poor diets. Future research should confirm whether and how irisin may interact with long-term diets to influence obesity.

\section{Caloric restriction does not influence cortisol levels}

To determine the role of weight loss in overweight individuals on cortisol levels, Tam et al. (11) implemented 24-week $25 \%$ calorie restriction with or without exercise in 35 otherwise healthy overweight adults (aged $38.1 \pm 5.8$ years) as compared to a control group of 11 participants (aged $38.5 \pm 7.4$ years) who were assigned to a weight maintenance diet. While diet changes overall caused $10 \%$ weight loss without impacting cortisol levels, decreased insulin sensitivity was correlated with elevated morning $(p<0.004)$ and diurnal $(p<0.02)$ cortisol levels. Future studies need to determine whether changes in insulin sensitivity may indirectly cause the changes in cortisol with weight loss in obesity and whether other aspects of weight loss, such as exercise, may also play a role in cortisol changes.

\section{Mediterranean diets, without weight loss, do not influence adipokines}

To determine how a Mediterranean diet may impact adipokine levels for individuals with metabolic syndrome, Richard et al. (12) examined 26 men between 24 and 62 years of age with metabolic syndrome. Participants consumed a North American diet for 5 weeks and then a Mediterranean diet for 5 weeks- both under isocaloric conditions, which they then followed with a 20-week caloric restriction period of weight loss and then a 5-week Mediterranean diet, again under isocaloric conditions. The Mediterranean diet prior to caloric restriction or weight loss did not impact leptin, resistin, adiponectin, or plasminogen activator inhibitor-1, but after caloric restriction and weight loss, it decreased both leptin by $32.1 \%$ and increased adiponectin by $14 \%$ compared with the control diet and by $30.4 \%$ and $12.9 \%$ respectively as compared to the Mediterranean diet before caloric restriction. Thus, without weight loss, a Mediterranean diet does not appear to impact most adipokines for individuals with metabolic syndrome. Future controlled studies should delve further into the usefulness and benefits of weight loss with a Mediterranean diet.

\section{Coffee influences insulin and glucose levels}

A common morning beverage, it is unclear how caffeinated coffee may alter blood sugar responses. Thus, Gavrieli et al. (13) conducted a randomized, controlled, cross-over study in 33 adults aged 19-44 years, of which 17 were overweight/obese and 16 were female, to determine how caffeinated coffee may impact postprandial glucose and insulin concentrations. The participants consumed coffee/placebo under three conditions of $200 \mathrm{~mL}$ water, coffee containing $3 \mathrm{mg} / \mathrm{kg}$ body weight caffeine, or coffee containing $6 \mathrm{mg} / \mathrm{kg}$ body weight caffeine. Coffee increased the area under the curve for glucose by $8.1 \%$ at the $3 \mathrm{mg} / \mathrm{kg}$ caffeine level and by $11.4 \%$ at the $6 \mathrm{mg} / \mathrm{kg}$ level. Coffee further delayed the rise of insulin in response to a standardized meal, suggesting that coffee may have effects on glucose/insulin that may impact the development of diabetes. Interestingly, these effects differed by gender and BMI, suggesting that women/men and obese/lean patients may respond differently to caffeinated coffee. Notably, these effects were short-lived and coffee only had a temporary effect on these outcomes. Future studies are needed to determine gender-specific mechanisms as well as whether coffee and/or caffeine contribute to these effects. 


\section{Effects of a short-term high fat diet and acute glucose ingestion on AMPK signaling}

AMP-activated protein kinase (AMPK), which senses energy and regulates metabolism, may be affected by consumption of a HFD. As immune peripheral blood mononuclear cells (PBMCs) are often activated by high-fat diets, Wan et al. (14) sought to determine in these cells the short-term dietary impact on AMPK and the mitochondrial oxidative phosphorylation (OXPHOS) proteins. Using nine healthy male adults (aged $21 \pm 3$ years) who participated in a seven-day HFD intervention, they were able to assess glucose tolerance with an oral glucose tolerance test (OGTT) as well as phosphorylation and circulating cytokines before and after the intervention. The results confirmed the hypothesis that AMPK signaling would decrease with a HFD. AMPK phosphorylation was even lower two hours later after OGTT when compared to following OGTT before the HFD. OXPHOS protein expression also was not affected. With the role that AMPK dysregulation may play in the pathogenesis of metabolic diseases, the study of PBMCs may allow for future studies to assess possible dietary effects on AMPK and inflammatory signaling.

\section{Sodium intake and overweight}

Song et al. (15) cross-sectionally examined the relationship between sodium intake and weight amongst 5955 participants (aged 19-64 years) who gave detailed dietary information. While men did show an increased risk of being overweight with higher sodium intake at an odds ratio of 1.37 and 1.67 for the fourth and fifth quintile of weight as compared to the lowest quintile, women showed only a marginally significant trend towards this same risk with an odds ratio of 1.31 from the highest as compared to the lowest quintile. Thus, this study suggests that increased sodium intake may relate to increased weight in adults, particularly in men. Future, well-controlled clinical trials would be needed to confirm these findings.

\section{Flaxseed and olive oil offer same benefits to inflammation}

Both olive oil and flaxseed oil appear to have anti-inflammatory effects, although they have different fat composition, and it is unclear whether one may have greater anti-inflammatory effects than the other. Kontogianni et al. (16) compared the effects of flaxseed and olive oil on lipid and inflammatory markers in a cross-over, randomized study of 37 normal weight, healthy young adults (aged 25.6 \pm 5.9 years) who typically adhered to a Mediterranean diet pattern. Flaxseed and olive oil showed no differences in adiponectin, tumor necrosis factora, CRP, lipid levels or glucose. However, they did show reduction in total by about $5 \%$ and low-density lipoprotein by about $6.7 \%$ cholesterol levels with both oils from baseline. Thus, while both oils are beneficial, there appear to be no significant differences in inflammatory or lipid benefits between olive or flaxseed oil. Future studies may wish to examine these oils in other dietary patterns, as well as in comparison to other common oils, such as vegetable or canola oil.

\section{Effects of fish or fish oil on adiponectin}

Whether a food item or supplements of the desired ingredient may have the same effect is a common question. Neale et al. (17) used a randomized trial to examine whether total or high molecular weight adiponectin may be changed in 29 overweight and obese participants who 
underwent four weeks of isocaloric dietary changes with either $1.8 \mathrm{~g}$ fish or fish oil supplement. High molecular weight adiponectin was significantly increased by about $8 \%$ in the group which consumed more fish, whereas it decreased by about $17 \%$ in the group which was given supplements. This study may indicate that the effects of fish on adiponectin are regulated by more than fish oil itself, and thus, that the supplements do not impart the same benefit. Future studies may seek to identify which is this beneficial component of fish.

\section{Effects of different types of caloric restriction on insulin signaling}

Wang et al. (18) set out to determine the differential effects of caloric restriction based on carbohydrate or fat restriction on body composition and insulin sensitivity. They performed euglycemic-hyperinsulinemic clamps and skeletal muscle biopsies of 18 obese individuals (20-45 years old) without diabetes after 5 days of a eucaloric diet, 5 days of a high fat/low carbohydrate diet, or 5 days of a low fat/high carbohydrate diet. While being on a $30 \%$ calorie restricted diet of either low fat/high carbohydrate or high fat/low carbohydrate did not differentially effect weight loss or whole-body insulin sensitivity, the low fat/high carbohydrate diet improved skeletal muscle insulin signaling in a way that would eventually improve insulin sensitivity by increased phosphorylation of IRS-1 tyrosine by about $60 \%$ and decreased phosphorylation of IRS-1 Ser307 by about 30\%, while the high fat/low carbohydrate diet led to opposite changes that may lead to insulin resistance by increasing phosphorylation of IRS-1 Ser307 by around 100\% and decreasing IRS- 1 associated p110 by about $25 \%$. Thus, the type of caloric restriction in dieting may be significant to change overall insulin sensitivity.

\section{Diet alone impacts cardiometabolic risk}

To better define the relationships between exercise, insulin resistance, and lipid accumulation, Rinnankoski-Tuikka et al. (19) examined in a controlled study high fat and low fat diets in 58 male C57BL/6J mice who were active or sedentary for 19 weeks. Another group of 30 mice, who were initially fed the HFD and were sedentary for 9 weeks, were then switched to a low fat diet while assigned to be sedentary or active for the remaining 10 weeks. The HFD increased triglycerides in muscle tissue by about 55\%. Switching to the low fat diet reversed these changes, and exercise- whether they were assigned to be sedentary or active- did not impact these effects. These results need to be repeated and confirmed in humans and the role of exercise in regulating insulin sensitivity and lipid accumulation better defined.

\section{Impacts of diet on fertility and reproductive function}

Adipose tissue has clear implications on female reproductive functions and fertility. In order to determine how obesity may impact ovarian follicle development, Wang et al. (20) examined with a controlled, randomized trial. 36 female rats (12 weeks old) who were fed a control, calorie restricted or HFD for 18 weeks. Those on the HFD showed less primordial follicles, decreased by about $49 \%$, and increased atretic follicles, by about $25 \%$, with increased levels of mTOR pathway proteins by about $40 \%$ and decreased levels of SIRT1 signaling proteins by about $25 \%$. The calorie restricted rats showed the opposite pattern with increased primordial follicles by about $44 \%$ and decreased atretic follicles by about $16 \%$, while mTOR signaling proteins decreased by about $20 \%$ and SIRT 1 increased by about 
$30 \%$. These results might indicate evidence of changes that are associated with early puberty and infertility during obesity, as seen with the HFD, while calorie restricted rats show changes associated with the opposite pattern of delayed puberty and prolonged fertility. Additionally, better understanding of how mTOR and SIRT1 influence these effects may lead to effective therapies to combat reproductive consequences of obesity.

\section{Exercise}

\section{Exercise impacts cardiometabolic function in type 2 diabetes}

Since type 2 diabetes can complicate cardiovascular health, there is a clear need to identify effective therapeutic strategies to slow non-ischemic subclinical left-ventricular (LV) dysfunction disease progression towards heart failure. Thus, Sacre et al. (21) examined in a controlled, randomized clinical trial if a six-month exercise intervention would improve peak oxygen uptake $\left(\mathrm{VO}_{2}\right.$ peak) and cardiac autonomic function in 49 subjects with type 2 diabetes and subclinical heart disease as compared to the standard of care (25 participants aged $60 \pm 9$ years in the control condition and 24 participants aged $59 \pm 10$ years in the exercise condition). The exercise intervention increased $\mathrm{VO}_{2}$ peak by $11 \%$ and decreased resting heart rate by about $3 \%$, indicating some significant improvements. Although myocardial fibrosis may have abated with physical activity, LV function failed to improve. Exercise training, therefore, has the ability to ameliorate exercise intolerance and improve some cardiovascular functions in these patients. Alterations to the current exercise design, whether in duration, intensity, or complementary therapies, could potentially shed more light on further benefits of exercise training in type 2 diabetic patients with subclinical LV dysfunction.

\section{Effects of acute or chronic exercise on irisin levels}

To test the differences between acute and chronic exercise on irisin levels, Huh et al. (22) examined irisin levels in 14 females (aged $24.3 \pm 2.6$ years) before, after 6 weeks of longterm whole-body vibration exercise training following an acute session of exercise, and outside of exercise but after 6 weeks of training. Irisin was significantly elevated immediately following the acute bought of exercise at 6 weeks (by about 91\%), but did not increase at the end of 6 weeks. This suggests that exercise consistently elevates irisin levels without changing baseline levels with chronic exercise. Future studies may want to examine other types of exercise for similar changes.

\section{KLAKS improves cardiometabolic risk}

To evaluate the success of Concept Leipzig: Adiposity therapy for school aged children (KLAKS), a one-year exercise-based lifestyle program for overweight/obese children, Bluher et al. (23) examined metabolic and anthropometric outcomes of 115 overweight or obese children (BMI greater than the $90^{\text {th }}$ percentile between the ages of 7 and 18) who participated in KLAKS. Indeed, KLAKS decreased metabolic risk factors, including body fat by about $11 \%$, BMI by about $6 \%$, and $\mathrm{HbA1c}$ by about $5 \%$, suggesting decreased risk of obesity and diabetes for children who participated in the KLAKS program. Future studies may examine how this program would influence other metabolic comorbidities of obesity, including cardiovascular disease risk. 


\section{Impacts of exercise breaks on long sedentary periods}

When sedentary behavior is uninterrupted, it may increase insulin resistance and glucose intolerance. Saunders et al. (24) sought to determine in a cross-over, interventional study if periodic light walking and/or moderate physical activity would improve these parameters in 19 children and adolescents ( 8 females; aged 10-14 years). All subjects participated in three conditions of 8 hours of sitting, 8 hours of sitting with 2 minute walking breaks every 20 minutes, and 8 hours of sitting with 2 minute walking breaks and two 20-minute moderate physical activity breaks. They found that breaking up a long sedentary period with either light walking or light walking and moderate exercise does not change the effects of a long sedentary period on insulin, glucose, or lipids. These results may suggest that other solutions would be needed to combat metabolic problems resulting from long periods of sedation. Future studies may want to test longer-term impacts of these different scenarios as well as other options for improving the impacts of long sedentary periods.

\section{Exercise mitigates some metabolic effects of a high fat diet}

Since exercise improves postprandial glucose and insulin, Numao et al. (25) tested whether this effect may be impacted by a high-fat diet, which typically impairs postprandial glucose and insulin. Thus, they performed a randomized, cross-over study in 11 healthy men (aged $27 \pm 1$ years) with 3 three-day interventions of a normal diet, a HFD, and a HFD with a single exercise session on the third day. They found that this single session of exercise did not reverse the impairment in postprandial glucose and insulin caused by a high-fat diet. On the other hand, exercise did normalize GLP-1, which had been increased by the high-fat diet. This study suggests that exercise may mitigate some but not all of the metabolic impairments caused by a high-fat diet. Future studies may seek to discover if these effects are further improved by longer or multiple exercise sessions.

\section{Irisin increases hippocampal neuronal growth at pharmacological doses}

Moon et al. (26) studied how irisin, a novel myokine, might influence hippocampal neurogenesis in vitro with H19-7 cell lines. Pharmacological (50-100nmol/L), but not physiological ( $5-10 \mathrm{nmol} / \mathrm{L})$, concentrations increased cell proliferation by $70-80 \%$ and signal transducer and activator of transcription 3 (STAT3) activation by $100 \%$ as compared to control. However, neither physiological nor pharmacological concentrations altered other markers of neurogenesis, including microtubule-associated protein 2 or synapsin. These data suggest that irisin may have limited effects on hippocampal neurogenesis. Future studies may want to investigate how irisin may behave in vivo and whether it may have effects on memory at pharmacological doses.

\section{Circulating irisin increases in response to exercise in pigs}

In order to determine if exercise might increase FNDC5 mRNA and protein, or plasma irisin in pigs, Fain et al. (27) examined muscle and adipose tissue from castrated male Rapacz familial hypercholesterolemic ( $\mathrm{n}=13 ; 10-11$ months of age) and Yucatan miniature $(\mathrm{n}=16$; 10-12 months of age) pigs after 16-20 weeks of exercise to look for these key markers using reverse transcription polymerase chain reaction and western blotting techniques in a cross-sectional study. They did not find increases in FNDC5 mRNA or protein in the muscle 
or adipose tissue of either of the pigs. However, circulating irisin, as measured by ELISA, was increased in these animals by $42 \%$ after exercise. Thus, the authors hypothesize that exercise in pigs may not be as good of a model as mice. Future research may want to investigate how/why the circulating irisin is elevated without changes in FNDC5 mRNA.

\section{Novel findings in type 2 diabetes and insulin sensitivity DPP4 treatment effects on inflammation}

Sitagliptin, a drug used in treatment of diabetes mellitus type 2, selectively inhibits dipeptidyl peptidase-4 (DPP4), a precursor of GLP-1. With the increasing presence of inflammation and endothelial dysfunction in atherosclerosis, which is often associated with type 2 diabetes, it is imperative to examine potential drug benefits with anti-inflammatory capabilities. Tremblay et al. (28) performed a double-blind, cross-over, placebo-controlled study in 36 adults with type 2 diabetes (aged $58.1 \pm 6.4$ years) using $100 \mathrm{mg} /$ day of sitagliptin or placebo for a 6-week period with a 4 week washout between phases. They discovered that sitagliptin significantly decreased inflammatory biomarkers such as CRP by $44.9 \%$, interleukin (IL)- 6 by $24.7 \%$ and IL- 8 by $7.8 \%$. Additionally, the increase in GLP-1 inversely correlated with the decrease in CRP $(\mathrm{p}<0.01)$. These improvements may indicate potential future usefulness of sitagliptin in managing other metabolic diseases. Future studies may want to test the usefulness of sitagliptin therapy on cardiovascular disease and obesity.

\section{Influences of estrogen and testosterone on insulin sensitivity}

Exposure to high levels of testosterone or estrogen in skeletal muscle has been found to decrease insulin sensitivity. To better understand how these hormones may influence insulin sensitivity, Garrido et al.(29) examined whether acute skeletal myotube exposure in vitro to supraphysiological doses of either estradiol or testosterone (concentrations for both: 0 , $10 \mathrm{nM}, 100 \mathrm{nM}, 1 \mu \mathrm{M}, 10 \mu \mathrm{M})$ directly affected glucose metabolism. Skeletal biopsies for cell culture were obtained from 19 healthy volunteers ( 9 male; aged $42 \pm 4$ years). Both $10 \mu \mathrm{M}$ estrogen and testosterone decreased glucose metabolism as represented by glucose transitioning to glycogen by 39 and $54 \%$ respectively as compared to a control case in the presence of $60 \mathrm{nM}$ insulin. Furthermore, this effect was dependent on protein kinase $\mathrm{C}$ (PKC) activation, and indeed, estradiol and testosterone caused a $41 \%$ and $106 \%$ increase, respectively, in phosphorylation of PKC. Since the PKC signaling pathways appear to be influenced by the aforementioned hormones, future studies should focus on the differing $\mathrm{PKC}$ isoforms to determine the exact mechanism through which each hormone regulates glucose metabolism and how these steroids may interact with glucose metabolism at physiological doses.

\section{Epigenetic changes relating to obesity and type 2 diabetes}

Epigenetic changes relating to immune function as it operates within obesity and type 2 diabetes in not yet clear. In order to better understand how DNA methylation may be changed in obesity and diabetes, Simar et al. (30) collected cross-sectional data from 44 male patients. Of those, 14 were obese, non-diabetics (aged $35.1 \pm 6.7$ years), 11 were agematched lean (aged $34.8 \pm 3.0$ years), 12 were type 2 diabetics (aged $44.1 \pm 6.5$ years) and 8 
were age-matched lean (aged $44.1 \pm 6.0$ years). Obesity and type 2 diabetes demonstrated increased methylation in B and natural killer cells of up to double the methylation, which increased with increasing insulin resistance as measured by HOMA-IR ( $<<0.009)$. Thus, it appears that epigenetic changes in lymphocytes may relate to changes in inflammation and immune cell function observed in obesity and diabetes. Future studies may seek to determine the directionality and underlying mechanisms of these changes.

\section{Epigenetic effects of a diabetic intrauterine environment}

To examine the role of DNA methylation in diabetes risk, del Rosario et al. (31) examined leukocytes from Pima Indian adolescents whose mothers had (14 participants; aged $15.5 \pm$ 5.1 years) or didn't have (14 participants; aged $16.2 \pm 5.8$ years) diabetes during pregnancy. DNA methylation was found to be different in the two groups for promoters of maturity onset diabetes of the young (MODY; hsa04950) and Notch signaling pathways (hsa04330). Thus, these changes may in fact increase risk for type 2 diabetes. Future studies should further investigate whether these epigenetic changes do in fact lead to later in life type 2 diabetes.

\section{Polymorphisms of the adiponectin gene in relation to microvascular complications among diabetics}

In order to determine if polymorphisms of the adiponectin gene may influence microvascular complications in type 2 diabetes, Choe et al. (32) genotyped 708 patients with type 2 diabetes and compared this with incidence of diabetic nephropathy, which was defined as urine albumin/creatinine ratio (UACR) greater than $30 \mathrm{mg} / \mathrm{g}$. Even when controlling for other possible confounders, the GG genotype at rs2241766 for the adiponectin gene was significantly associated with diabetic nephropathy with an odds ratio of 1.96 but not retinopathy or neuropathy, implicating this polymorphism with the specific risk for diabetic nephropathy. Thus, patients with type 2 diabetes and the GG genotype at rs2241766 should be carefully monitored for diabetic nephropathy. Future studies may want to better define the role of this gene in diabetic nephropathy.

\section{Independent effects of diabetes and aging on incretins}

By examining middle-aged as compared to older individuals with and without type 2 diabetes, Geloneze et al. (33) attempted to determine whether and how diabetes and aging may interact to alter insulin sensitivity and incretins. They examined cross-sectionally 25 middle-aged participants (aged $45 \pm 3$ years), of which 13 had type 2 diabetes, as compared to 25 older adults (aged $65 \pm 2$ years), of which 13 had diabetes. Both middle-aged and older adults with diabetes had decreased insulin sensitivity index (ISI) by about $70 \%$ as compared to non-diabetics. GLP- 1 was reduced in the older diabetics at $p<0.05$. These results may show an increased impact of aging with diabetes on incretins (GLP-1), but this will need to be confirmed in future studies.

\section{Effects of estrogen on insulin sensitivity}

How obesity and estrogen deficits may interact to impact insulin sensitivity in the brain is not yet clear, although obesity alone reduces insulin sensitivity and estrogen therapy may 
improve these deficits. Pratchayasakul et al. (34) examined how 30 female rats (about 6-7 weeks old) given a 12 week HFD or control diet, who did or did not receive ovariectomies, were impacted by subsequent $50 \mu \mathrm{g} / \mathrm{kg}$ estrogen replacement or placebo therapy from week 13 for 30 days. All ovariectimized rats had impaired insulin sensitivity as measured by HOMA index, but only rats that ate the control diet showed improved insulin sensitivity with estrogen replacement back to control levels. Thus, there may be a greater impact of combined obesity and estrogen deprivation on insulin sensitivity that will need to be further defined and more effective treatments identified.

\section{Inflammatory effects of obesity impacting insulin sensitivity}

While many obese individuals develop type 2 diabetes, others do not. To determine differences in inflammation that may underlie these differences, van Beek et al. (35) compared obese women who had diabetes (28 participants; aged $51 \pm 7$ years) or were insulin-sensitive (26 participants; aged $48 \pm 6$ years) to 12 lean controls (aged $50 \pm 5$ years). As predicted, those who were both obese and diabetic showed higher levels of inflammatory markers, including IL-6 (increased by about $283 \%$ in insulin-sensitive obese as compared to lean and by about $556 \%$ in diabetic obese as compared to lean), increased leukocytes by about $18 \%$ in obese diabetics as compared to insulin-sensitive, and increased circulating $\mathrm{T}$ cells by about $40 \%$ in obese diabetics as compared to insulin-sensitive obese. These results suggest that inflammation may underlie the changes from insulin sensitive to resistant obesity, though this will need to be confirmed with other studies and more in-depth experiments.

\section{Effects of insulin sensitivity/resistance on sleep}

Although pre-diabetes, type 2 diabetes and obesity have been associated with short sleep duration, how insulin resistance may impact sleep is unclear. Liu et al. (36) examined crosssectionally by self-report how much obese individuals who were insulin-sensitive (21 participants; 8 men; aged $55 \pm 9$ years) or insulin-resistant ( 35 participants; 15 men; aged 56 \pm 9 years), but not diabetic, reported sleeping on average, where under seven hours per night was considered to be of short duration. Indeed, they confirmed that insulin-resistant obese individuals had shortened sleep duration by about $10 \%$ as compared to the insulin-sensitive obese individuals, despite no differences in BMI or waist circumference. The authors conclude that there is an independent association between insulin resistance and shortened sleep duration without diabetes. Future, controlled studies should be done to confirm these findings.

\section{Diabetes and prediabetes have important impacts on coronary flow reserve}

Coronary flow reserve can be used to establish the existence of coronary artery disease. Erdogan et al. (37) sought to compare coronary flow reserve in 65 prediabetics (aged $51.4 \pm$ 8.6 years) to that in 45 individuals with (aged $51.6 \pm 7.2$ years) or 43 individuals without (aged $50.4 \pm 8.5$ years) diabetes in order to determine risk for coronary artery disease. Coronary flow reserve was lowest in individuals with diabetes, at about $22 \%$ of individuals without diabetes, but still lower in prediabetics than controls by about $13 \%$, even though age, gender, and smoking status were matched across groups. Additionally, coronary flow reserve inversely correlated with age $(\mathrm{p}<0.04)$, fasting glucose levels $(\mathrm{p}<0.001)$, and HbA1c 
levels ( $\mathrm{p}<0.001$ ), suggesting a potential association between coronary flow reserve and diabetes. Thus, this study suggests that prediabetics should also be monitored for coronary artery disease. Future, longitudinal and prospective studies should confirm these findings.

\section{Biomarkers for cardiometabolic dysfunction Cytokines associated with cardiometabolic risk}

With much yet to be determined about the cytokines irisin and omentin-1, Panagiotou et al. (38) aimed to investigate if these markers may be associated with cardiovascular risk and lipoprotein subparticles. The effect of alcohol consumption on these markers was examined in 39 subjects with either diabetes or two other cardiovascular risk factors cross-sectionally at baseline, 3 months and 6 months. Repeated measures from baseline to 6 months exhibited an inverse correlation between decreasing irisin and increasing omentin-1 with a $7.4 \%$ irisin decrease per 1-standard deviation increment in omentin-1. In addition, after adjusting for age, sex and race, a decrease in irisin was observed with increasing high density lipoprotein (HDL) levels with a $7.3 \%$ decrease per a $10 \mathrm{mg} / \mathrm{dL}$ increment. Omentin-1 and mean very low density lipoproteins (VLDL) size were positively associated with a $3.8 \%$ increase in omentin-1 per a 1-SD increment of VLDL. Both cytokines show promise as cardiovascular risk markers. Due to potential irisin resistance findings in those at risk for metabolic syndrome, future studies are warranted both for possible irisin sensitizers such as omentin-1, as well as for aims to explore if a direct mechanism exists between irisin and omentin-1.

\section{Relationship between adiponectin and stroke}

Whether adiponectin may play a role in stroke is unclear. To better clarify if and how adiponectin may interact with ischemic stroke, Kuwashiro et al. (39) studied adiponectin levels from 171 patients (115 male; aged $68.3 \pm 10.1$ years) and 171 matched controls (115 male; aged $68.1 \pm 10.1$ years) at stroke onset and at $3,7,14$, and 90 days following. Overall, at stroke onset, adiponectin did not significantly vary between all stroke patients and controls, although it was lower for patients with atherothrombotic infarction by about $29 \%$ and higher in patients with cardioembolic infarction by about 39\%. Adiponectin levels at stroke onset were higher in patients with poorer functional outcomes by about $43 \%$. These results may indicate some relationships between adiponectin and stroke, particularly certain subtypes, but this will need to be confirmed and explored in future studies.

\section{Actions of adiponectin in inflamed adipose tissue}

To better understand the role of adiponectin in adipocytes, Nakatsuji et al. (40) set out to determine whether adiponectin was present in adipose tissue from male lean (wild type) or obese $(d b / d b)$ mice ( $n=4-6$, each group). Adiponectin was present in adipose mature adipocyte and stromal vascular tissues and was higher in obese as compared to lean mice by about $300-400 \%$. When adiponectin was given to mice that were knock-outs for adiponectin, adiponectin collected in adipose tissue, particularly when obese. This study suggests that adiponectin may selectively accumulate in adipose tissues during obesity. Further research should look into mechanisms and whether and how this may relate to observed decreased overall levels of adiponectin in obesity. 


\section{Adioponectin may improve adipose tissue vascularity in obesity}

Aprahamian (41) examined how adiponection-deficient (apn-KO) and over-expressing (apnTG) mice reacted to a chow or high fat/high sucrose diet for 32 weeks starting at 8 weeks of age as compared to wild type in order to determine how adiponectin may influence the adipose tissue microenvironment. Over-expression of adiponectin increased vascularity and perfusion of adipose tissue by about $200 \%$ as measured by staining with green for vasculature and measuring the luminosity which indicated capillary density. Adiponectin deficient mice did not show poorer perfusion or function and with the high fat/high sucrose diet, they did not show impaired insulin resistance or hepatic steatosis as compared to wild type. Aprahamian concludes that increased adiponectin may improve conditions of obesity by increasing adipose tissue vascularity and perfusion. The signaling pathways involved should be elucidated to better demonstrate how adiponectin may influence/improve obesityrelated inflammation.

\section{Markers of liver function also mark metabolic health}

To examine how markers of liver function related to overall metabolic health, Liu et al. (42) examined data from a cross-sectional study of 616 randomly enrolled participants (aged 18.3 \pm 0.02 years) and prospective cohort of 93 subjects who came back 2 years later. Crosssectionally, alanine transaminase (ALT) and gamma-glutamyltransferase (GGT) were correlated with cardiometabolic outcomes including heart rate, systolic and diastolic blood pressure, cholesterol, high density lipoproteins, low density lipoproteins, triglycerides, and dyslipidemia, while fetuin-A was correlated with insulin, insulin resistance, and blood pressure. In the prospective arm, ALT and GGT were found to predict anthropometric outcomes at 2 years with multivariable linear regression models. These results may suggest that traditional liver markers, such as ALT and GGT, would be important to monitor for resultant overall metabolic health. Future studies should confirm and expand on these findings.

\section{Irisin may be a marker for fatty liver disease}

In order to determine whether irisin levels may be a marker of fatty liver, Polyzos et al. (43) cross-sectionally examined irisin levels in 31 patients (aged 53.9 \pm 2.9 years) with biopsyconfirmed simple steatosis or steatohepatitis (NASH) as compared to 24 lean (aged $54.2 \pm$ 1.6 years) and 28 obese (aged $52.6 \pm 1.6$ years) controls without non-alcoholic fatty liver disease (NAFLD). Irisin levels were decreased with obesity by about $32 \%$ as compared to lean controls and both simple steatosis by about $39 \%$ and NASH by about $28 \%$ as compared to lean adults, which remained after adjustment for BMI, gender, age, exercise, and insulin resistance. Irisin was also associated with portal inflammation as irisin was about $20 \%$ higher in those with portal inflammation. Thus, there appears to be links between irisin levels and fatty liver, though these will need to be confirmed with future clinical studies.

\section{Irisin as a novel marker for future insulin resistance}

In order to determine if irisin may mediate the relationship between weight regain after loss and insulin resistance, Crujeiras et al. (44) examined irisin levels in 136 obese participants (62 women; aged $43.0 \pm 11.2$ years) who had undergone an eight-week weight loss program 
of $30 \%$ reduced energy. Irisin levels were measured just before starting the program, immediately following the program, and after a four to six month follow-up period, during which half had regained weight. Increased insulin resistance, measured by HOMA-IR, at follow-up was associated with elevated irisin levels at baseline, before the weight loss program $(\mathrm{p}<0.05)$. This finding may suggest that irisin could be a marker for insulin resistance upon weight regain. The mechanisms which may mediate this association need to be delineated in future studies.

\section{Irisin mediates energy expenditure in a subset of humans}

In order to determine whether responsiveness to irisin may mediate differences in energy expenditure, Swick et al. (45) monitored irisin levels over the course of a day in 17 postmenopausal women between 50 and 70 years of age as they were sedentary and consumed standardized meals. Irisin levels did not overall vary with individual requirements, although in a subset of participants whose energy expenditure was greater than what would be expected based on fat free mass, irisin levels, but not leptin or adiponectin, were correlated with energy expenditure over the course of the day $(\mathrm{p}<0.0016)$. Therefore, it appears that there are individual differences in responsiveness to irisin that may modulate its effects. Future studies should delve further into these potential relationships in larger, controlled trials.

\section{Leptin does not influence fetuin-A, a marker of fatty liver}

Since fetuin-A appears to be important in fatty liver diseases, Hwang et al. (46) hypothesized that fetuin-A levels may be mediated by leptin. In a series of experiments examining fetuin-A levels with hypoleptinemic adults who were given metreleptin therapy, they attempted to parcel apart this relationship. The first substudy contained 15 lean adults ( 8 men aged $23.3 \pm 1.2$ years and 7 women aged $23.7 \pm 1.5$ years) and was a placebocontrolled, cross-over study with metreleptin therapy given at doses of $0.04 \mathrm{mg} / \mathrm{kg} / \mathrm{day}$ for men and $0.08 \mathrm{mg} / \mathrm{kg} / \mathrm{day}$ for women on day 1 which was increased for days 2-3 to 0.1 $\mathrm{mg} / \mathrm{kg} /$ day for men and $0.2 \mathrm{mg} / \mathrm{kg} /$ day for women. Measurements were obtained on day 1 and day 4 in the morning. The second substudy involved seven lean women (aged 25.0 2.2 years) who participated in an open label clinical trial of metreletin at doses of 0.08 $\mathrm{mg} / \mathrm{kg} / \mathrm{day}$ for $2 \mathrm{months}$ and then $0.2 \mathrm{mg} / \mathrm{kg} / \mathrm{day}$ for a third month. Measurements were taken one month before starting the study and after 1,3, 7, and 11 weeks of therapy. The third substudy involved 17 lean, hypoleptinemic women (aged 26.1 \pm 3.9 years), who participated in a randomized, placebo-controlled trial of metreleptin at doses of 0.08 $\mathrm{mg} / \mathrm{kg} /$ day for 12 weeks and then an increase to $0.12 \mathrm{mg} / \mathrm{kg} / \mathrm{day}$ if they had not yet responded. If weight decreased by more than 5\%, the metreleptin dose was decreased to 0.04 $\mathrm{mg} / \mathrm{kg} / \mathrm{day}$. Measurements were taken before and after 12, 24, and 36 weeks of therapy. The fourth substudy was an in vitro study using human hepatoma HepG2 cells that were treated with $0,5,10,20$, and $100 \mathrm{ng} / \mathrm{mL}$ leptin and then underwent real time PCR to examine them for fetuin-A mRNA expression. Fetuin-A was not moderated by caloric deprivation or metreleptin therapy in hypoleptinemia. Leptin given to cells did not modify fetuin-A mRNA expression. Therefore, it appears that leptin does not influence fetuin-A levels. Future studies may seek to determine whether and how fetuin-A modulates fatty liver disease outcomes. 


\section{Leptin improves mitochondrial function and hepatic steatosis in obese mice}

In order to determine how leptin influences mitochondrial function, Holmstrom et al. (47) treated obese and lean mice (aged 14-20 weeks) with daily $0.9 \%$ saline or $1 \mathrm{mg} / \mathrm{kg}$ body weight of leptin intraperitoneal injections for 5 days before extracting liver and muscle tissues to assess mitochondrial respiration. Obese mice showed decreased liver mitochondrial respiration by about $30 \%$ and increased muscle mitochondrial respiration by about $35 \%$, but this was corrected to control levels with leptin treatment. Leptin treatment in ob/ob mice also decreased liver weight by $32 \%$ as well as triglyceride content by $31 \%$. These findings suggest that leptin positively impacts mitochondrial function and hepatic outcomes in obesity. Future, controlled studies in humans should be performed to determine if leptin therapy may also show beneficial effects on hepatic and mitochondrial outcomes.

\section{Impact of leptin on the immune system}

In a recent in vitro study, Cassano et al.(48) revealed that leptin directly regulates $\mathrm{T}$ cell autophagy using human CD4(+)CD25(-) conventional T cells. During T cell receptor (TCR) stimulation, in vitro treatment with leptin inhibited autophagy through the mTOR pathway, and this inhibition depended on both duration and dosage. This finding was further confirmed in leptin-deficient mice that had higher than normal levels of autophagy. These results have important implications on leptin's role in nutritional status and immune activation, and these relationships should be explored more in the future.

\section{Adipokines in relation to myelodysplastic syndrome risk}

In order to determine how adipokines may relate to myelodysplastic syndrome, Dalmaga et al. (49) implemented a case-control study on 101 individuals with myelodysplastic syndrome (58-83 years old; 59 males) and 101 age- and gender-matched (57-83 years old; 59 males) control participants. The risk of myelodysplastic syndrome was associated with increased fetuin-A by about $10 \%$, decreased adiponectin by about $28 \%$, and decreased leptin by about $8 \%$, even when controlled for demographic and social variables, BMI, and insulin. These links should be better defined and a potential causal role between these adipokines and myelodysplastic syndrome should be investigated.

\section{Chemerin is not associated with acute coronary syndrome}

Coronary artery disease severity correlates with levels of the novel adipokine, chemerin, suggesting that this could be an indicator of heart disease. Aronis et al. (50) sought to discover if chemerin also predicts acute coronary syndrome. In a cross-sectional study of 90 men whose serum was collected 2 years prior to acute coronary syndrome and 162 matched controls (aged 66.3 \pm 9.6 years). Chemerin was unable to predict acute coronary syndrome, suggesting that chemerin may not serve as a general marker of heart disease though it may play a role in more chronic heart disease. Future studies should parcel apart this relationship and determine what role chemerin may play in relation to coronary artery disease.

\section{FGF21 in human brown adipose tissue}

In order to discover whether fibroblast growth factor 21 (FGF21) may target human brown adipose tissue, Hondares et al. (51) examined cross-sectional FGF21 gene expression in 
induced brown adipose tissue from nine adults with pheochromocytoma, ten adult controls, and 18 newborns (20-40 gestational weeks) who had died between 1995-2006 and were autopsied 2-3 hours after death. FGF21 mRNA was expressed in brown adipose tissue from infants (median $6.9 \times 10^{-8}$ ) and adults with pheochromocytoma (median $3.3 \times 10^{-8}$ ), but not control adult white adipose tissue, suggesting that FGF21 targets brown adipose tissue.

Future studies should determine the role FGF21 may play in interacting with brown adipose tissues.

\section{Cardiometabolic risk factors and interventions}

\section{Impact of weight loss surgery on metabolic comorbidities in adolescents}

Although weight loss surgery improves outcomes in adults, whether these bariatric surgeries may also improve metabolic comorbidities in adolescents remains to be discovered. Oberbach et al. (52) piloted a study in 10 obese adolescents (9.7-19.7 years of age) who underwent either laparoscopic sleeve gastrectomy or laparoscopic Roux-en-Y-gastric bypass bariatric surgery as compared to 17 normal-weight, age-and gender-matched adolescents (12.5-16.5 years of age) pre- and 12 months post-operatively. They discovered that after a year, risk factors for metabolic comorbidities, including diabetes (HOMA-IR decreased $51.9 \%$ ), cardiovascular disease (systolic blood pressure decreased 6.8\%, high density lipoproteins increased $21.4 \%$ ), and liver function tests (alanine aminotransferase decreased $43.9 \%$ ), were all improved. This study suggests that bariatric surgery may be sufficient to improve metabolic comorbidities in obese adolescents, though this will need to be confirmed with future studies.

\section{Childhood adversities influence cardiometabolic risk in midlife}

To examine how overall childhood adversity may confer obesity later in life, Davis et al. (53) performed a cross-sectional analysis of data from 210 midlife adults (aged $45.8 \pm 3.3$ years). Overall childhood adversity, which combined the number, severity, and chronicity of adversities during childhood, was a significant predictor of central obesity as measured by waist-hip ratio with multivariate linear regressions, but not BMI. Thus, this study suggests that overall childhood adversity may represent a unique metabolic risk in the form of central obesity in midlife. These findings may have implications of healthcare as these individuals may be further at risk for other metabolic dysfunctions, though this will need to be confirmed by future studies.

\section{Stress during adolescence impacts metabolic risk differentially by gender}

Stress causes changes in weight that can lead to obesity, but it is not clear exactly how these changes may occur. Krolow et al. (54) assessed with a randomized, placebo-controlled study how isolation stress during adolescence (beginning on postnatal day 21 until 28) may alter diet, weight gain, and hormones in both male and female Wistar rats. Rats were divided into four groups of which 31 controls received lab chow, 36 controls received palatable diets, 36 isolated animals received lab chow, and the remaining 36 isolated animals received palatable diets. After postnatal day 28, rats were returned to normal cage groupings and fed standard lab chow. In both females and males, isolation stress led to increased consumption of a palatable diet at levels up to double the kcal of the chow diet under the same isolation stress 
and at levels more than the kcal consumed of the palatable diet in control situations. In females, this also increased weight gain by about $30 \%$ over the chow in the same stress situation, adiponectin by about $77 \%$ over the palatable diet in control situations, and triacylglyceride levels by $66 \%$ over the palatable diet in control situations. In males, stress increased hypothalamic neuropeptide Y levels by up to $467 \%$ over the rats who were not exposed to stress. Although in females, these effects disappeared in adulthood, males had increased weight over time after exposure to the palatable diet over the chow diet during stress and decreased adiponectin by about $20 \%$ over the control group who had also received the palatable diet. Thus, these findings demonstrated differential effects of stress in adolescence with females showing short-term feeding effects and males showing longerterm effects in adulthood. Future research should endeavor to identify potential mechanisms underlying these changes.

\section{Timing of menarche may predict risk of metabolic syndrome}

In order to find more predictors for the risk of metabolic syndrome, Glueck et al. (55) conducted a 26-year prospective follow-up study of 272 girls (aged 5-22 years at first study and 30-46 years at follow-up) to determine how early ( $\leq 10$ years) and late ( $\geq 16$ years) menarche may impact the risk of adult metabolic syndrome. Early menarche was associated with high childhood BMI $\left(21.2 \pm 1.0 \mathrm{~kg} / \mathrm{m}^{2}\right)$ and increased risk of both childhood $(15 \%)$ and adult (36\%) metabolic syndrome. Late menarche was associated with low childhood BMI $\left(18.1 \pm 1.0 \mathrm{~kg} / \mathrm{m}^{2}\right)$ and increased incidence of adult metabolic syndrome (47\%). Metabolic syndrome in adulthood was also associated with early-late menarche, showing a u-shaped relationship. Thus, girls who have early or late menarche should be monitored carefully into adulthood for metabolic syndrome. Future studies may seek to investigate further potential causal relationships between early or late menarche with obesity and metabolic syndrome.

\section{Methionine restriction decreases risk of hepatic steatosis in obesity}

Malloy et al. (56) conducted a randomized, placebo-controlled trial to investigate how a 14week dietary methionine restriction $(0.12 \%$ methionine) might decrease hepatic steatosis in 10 -week-old obese $o b / o b$ mice as compared to a control diet ( $0.86 \%$ methionine). Methionine restriction reduced body weight by about $18 \%$, decreased liver triglycerides by about 50\%, decreased insulin and HOMA-IR by about 50\%, reduced the gene expression of inflammatory markers such as tumor necrosis factor-a by about $46 \%$ and CCR 2 by about $44 \%$, increased very low density lipoprotein secretions by about $20 \%$, and increased adiponection levels by about $129 \%$. Therefore, the authors concluded that methionine restriction may decrease steatosis by increasing lipid export and reducing inflammatory responses to obesity. Future randomized, controlled trials should be conducted in humans to confirm these findings.

\section{GLP-1 therapies may decrease cardiometabolic risks}

GLP-1 analogues have gained attention for their effective treatment of diabetes and obesity, although they may also have important cardioprotective effects as well. Aronis et al. (57) evaluated GLP-1 effects (escalating doses at 50-2000 nmol/L) on angiogenesis in vitro with human umbilical vein endothelial cells, and discovered that GLP-1 does increase 
angiogenesis from $200 \mathrm{nmol} / \mathrm{L}$ to $1000 \mathrm{nmol} / \mathrm{L}$ with an inverted $\mathrm{u}$-shaped dose response curve and the optimal dose being at $500 \mathrm{nmol} / \mathrm{L}$. Treating the system with inhibitors for Akt, PKC, or src pathways decreased the effects of GLP-1 on angiogenesis, suggesting that its cardiovascular effects are mediated through these pathways. Future research should better delineate these pathways and interactions.

\section{New techniques}

\section{Non-invasive MRI technique to study brown adipose tissue}

In an effort to develop a new technique to classify brown as compared to white adipose tissue, Holstila et al. (58) compared the usual positron emission tomography (PET) with 18fluorodeoxyglucocose $\left({ }^{18} \mathrm{FDG}-\mathrm{PET}\right)$ technique with a new dual echo spectral presaturation inversion recovery (DUAL-SPIR) magnetic resonance imaging (MRI) scan with eleven male Sprague-Dawley rats (weighing 325-400g). The new DUAL-SPIR MRI was more accurate, meaning a better correlation, in comparison to histological findings $(\mathrm{p}<.017)$ than the standard PET technique ( $\mathrm{p}<.078)$ and can be applied to human subjects. The DUALSPIR MRI under normal temperature conditions was also feasibility tested in one male and two female normal-weight human subjects as compared to the traditional PET method after cold exposure. DUAL-SPIR MRI measured 51, 32, and $53 \mathrm{~g}$ of brown adipose tissue in each of the cases, while PET measured 54, 46, and $16 \mathrm{~g}$ respectively for each of the cases. Therefore, there is potential for a non-invasive method to more accurately measure brown adipose tissue in humans using this type of MRI. Future, larger human studies should confirm these preliminary findings.

\section{New MRI techniques to examine hepatic steatosis}

In order to test a method for early detection of hepatic steatosis, d'Assignies et al. (59) examined how multi-echo MRI, MR spectroscopy (MRS), and histology ex vivo postmortem may be able to detect steatosis in 32 rats (13 8-week-old Wistar, 6 26-week-old Wistar and 13 diabetic, 8-week-old Goto-Kakizaki rats) who were given a 72-hour intravenous infusion of glucose and intralipid fat emulsion or saline. MRI and MRS detected liver steatosis showed greater sensitivity while maintaining accuracy (sensitivity of $92.9 \%$ vs. $50 \%$ in histology and area under the ROC curve of 0.841 vs. 0.641 for histology) of which rats received the glucose and intralipid infusion as compared to placebo. The amount of fat shown in these imaging techniques correlated with insulin and c-peptide levels (all $\mathrm{p}<$. 01). Thus, these non-invasive magnetic resonance techniques for imaging should be tested both in vivo in rats to ensure that these ex vivo results are reproducible in vivo and then in humans to see if they may also be effective at early detection of liver steatosis.

\section{Uptake of 18-fluorodeoxyglucose assessed by CT and PET imaging to assess brown adipose tissue is not altered by hypoglycemia}

Since there is an association between hypoglycemia and both increased heat production and hypothermia, Schopman et al. (60) sought to determine if brown adipose tissue, which is activated by cold exposure and stimulated by the sympathetic nervous system, was also affected by hypoglycemia. Using PET and computer tomography to measure the uptake of labeled glucose analogue ${ }^{18} \mathrm{~F}$-fluorodeoxyglucose $\left({ }^{18} \mathrm{FDG}-\mathrm{PET}\right)$ in brown adipose tissue due 
to thermogenesis, they examined in nine healthy adults (aged 19-30 years old) the effect of hypoglycemia (blood glucose $2.5 \mathrm{mmol} / \mathrm{L}$ ) versus euglycemia $(4.5 \mathrm{mmol} / \mathrm{L})$ on brown fat. Under conditions of mild cold exposure $\left(17^{\circ} \mathrm{C}\right)$, it was concluded there was no differential effect of hypoglycemia on brown adipose tissue as compared to normal blood glucose levels. Future studies should determine a clearer relationship between hypoglycemia and increased energy expenditure, and which potential factors could be responsible.

\section{Conclusions}

While the field of metabolic-related research continues to grow and expand, we have gained much knowledge and insight into impacts of diet, exercise, biomarkers, risk factors, and potential new techniques to be employed in the future. These new insights have important effects on both clinical care and daily life, which is part of what makes metabolic-related research a critically growing field. Additionally, this wealth of new information will be a key foundation from which future research may continue to expand and grow.

\section{Acknowledgments}

Funding: NIH 5T32HD052961

Olivia M. Farr is supported by a NICHD 5T32HD052961.

\section{Abbreviations}

GLP-1 glucagon-like peptide 1

250HD 25-hydroxyvitamin D

RDA recommended daily allowance

IGF-1 insulin-like growth factor 1

HDL high density lipoprotein

HOMA-IR homeostatic model assessment of insulin resistance

HbA1c glycated hemoglobin

HFD high fat diet

BMI body mass index

AMPK AMP-activated protein kinase

PBMCs peripheral blood mononuclear cells

OXPHOS oxidative phosphorylation

OGTT oral glucose tolerance test

LV left ventricular

$\mathbf{V O}_{2}$ peak peak oxygen uptake

KLAKS Concept Leipzig: Adiposity therapy for school aged children 


$\begin{array}{ll}\text { STAT3 } & \text { signal transducer and activator of transcription } 3 \\ \text { DPP-4 } & \text { dipeptidyl peptidase-4 } \\ \text { IL } & \text { interleukin } \\ \text { PKC } & \text { protein kinase C } \\ \text { MODY } & \text { maturity onset diabetes of the young } \\ \text { ISI } & \text { insulin sensitivity index } \\ \text { VLDL } & \text { very low density lipoproteins } \\ \text { ALT } & \text { alanine transaminase } \\ \text { GGT } & \text { gamma-glutamyl transferase } \\ \text { NAFLD } & \text { non-alcoholic fatty liver disease } \\ \text { NASH } & \text { non-alcoholic steatohepatitis } \\ \text { TCR } & \text { T cell receptor } \\ \text { FGF21 } & \text { fibroblast growth factor 21 } \\ \text { PET } & \text { positron emission tomography } \\ \text { DUAL-SPIR } & \text { dual echo spectral presaturation inversion recovery } \\ \text { MRI } & \text { magnetic resonance imaging } \\ \text { MRS } & \text { magnetic resonance spectroscopy }\end{array}$

\section{References}

1. Goss AM, Chandler-Laney PC, Ovalle F, Goree LL, Azziz R, Desmond RA, et al. Effects of a eucaloric reduced-carbohydrate diet on body composition and fat distribution in women with PCOS. Metabolism: clinical and experimental. 2014 Epub 2014/08/16. 10.1016/j.metabol.2014.07.007

2. Liu C, Hu MY, Zhang M, Li F, Li J, Zhang J, et al. Association of GLP-1 secretion with antihyperlipidemic effect of ginsenosides in high-fat diet fed rats. Metabolism: clinical and experimental. 2014 Epub 2014/07/26. 10.1016/j.metabol.2014.06.015

3. Kampmann U, Mosekilde L, Juhl C, Moller N, Christensen B, Rejnmark L, et al. Effects of 12weeks high dose vitamin D3 treatment on insulin sensitivity, beta cell function, and metabolic markers in patients with type 2 diabetes and vitamin D insufficiency - a double-blind, randomized, placebocontrolled trial. Metabolism: clinical and experimental. 2014; 63(9):1115-24. Epub 2014/07/22. 10.1016/j.metabol.2014.06.008 [PubMed: 25044176]

4. Kondo K, Morino K, Nishio Y, Kondo M, Nakao K, Nakagawa F, et al. A fish-based diet intervention improves endothelial function in postmenopausal women with type 2 diabetes mellitus: a randomized crossover trial. Metabolism: clinical and experimental. 2014; 63(7):930-40. Epub 2014/05/23. 10.1016/j.metabol.2014.04.005 [PubMed: 24850465]

5. Henning PC, Margolis LM, McClung JP, Young AJ, Pasiakos SM. High protein diets do not attenuate decrements in testosterone and IGF-I during energy deficit. Metabolism: clinical and experimental. 2014; 63(5):628-32. Epub 2014/03/20. 10.1016/j.metabol.2014.02.007 [PubMed: 24641883]

6. Melo AM, Benatti RO, Ignacio-Souza LM, Okino C, Torsoni AS, Milanski M, et al. Hypothalamic endoplasmic reticulum stress and insulin resistance in offspring of mice dams fed high-fat diet during pregnancy and lactation. Metabolism: clinical and experimental. 2014; 63(5):682-92. Epub 2014/03/19. 10.1016/j.metabol.2014.02.002 [PubMed: 24636055] 
7. Silver HJ, Kang H, Keil CD, Muldowney JA 3rd, Kocalis H, Fazio S, et al. Consuming a balanced high fat diet for 16 weeks improves body composition, inflammation and vascular function parameters in obese premenopausal women. Metabolism: clinical and experimental. 2014; 63(4): 562-73. Epub 2014/02/25. 10.1016/j.metabol.2014.01.004 [PubMed: 24559846]

8. Wu L, Piotrowski K, Rau T, Waldmann E, Broedl UC, Demmelmair H, et al. Walnut-enriched diet reduces fasting non-HDL-cholesterol and apolipoprotein $\mathrm{B}$ in healthy Caucasian subjects: a randomized controlled cross-over clinical trial. Metabolism: clinical and experimental. 2014; 63(3): 382-91. Epub 2013/12/24. 10.1016/j.metabol.2013.11.005 [PubMed: 24360749]

9. Pham NM, Nanri A, Kochi T, Kuwahara K, Tsuruoka H, Kurotani K, et al. Coffee and green tea consumption is associated with insulin resistance in Japanese adults. Metabolism: clinical and experimental. 2014; 63(3):400-8. Epub 2013/12/18. 10.1016/j.metabol.2013.11.008 [PubMed: 24342074]

10. Park KH, Zaichenko L, Peter P, Davis CR, Crowell JA, Mantzoros CS. Diet quality is associated with circulating C-reactive protein but not irisin levels in humans. Metabolism: clinical and experimental. 2014; 63(2):233-41. Epub 2013/12/10. 10.1016/j.metabol.2013.10.011 [PubMed: 24315778]

11. Tam CS, Frost EA, Xie W, Rood J, Ravussin E, Redman LM. No effect of caloric restriction on salivary cortisol levels in overweight men and women. Metabolism: clinical and experimental. 2014; 63(2):194-8. Epub 2013/11/26. 10.1016/j.metabol.2013.10.007 [PubMed: 24268369]

12. Richard C, Royer MM, Couture P, Cianflone K, Rezvani R, Desroches S, et al. Effect of the Mediterranean diet on plasma adipokine concentrations in men with metabolic syndrome. Metabolism: clinical and experimental. 2013; 62(12):1803-10. Epub 2013/09/03. 10.1016/ j.metabol.2013.07.012 [PubMed: 23993420]

13. Gavrieli A, Fragopoulou E, Mantzoros CS, Yannakoulia M. Gender and body mass index modify the effect of increasing amounts of caffeinated coffee on postprandial glucose and insulin concentrations; a randomized, controlled, clinical trial. Metabolism: clinical and experimental. 2013; 62(8):1099-106. Epub 2013/03/19. 10.1016/j.metabol.2013.02.003 [PubMed: 23498899]

14. Wan Z, Durrer C, Mah D, Simtchouk S, Robinson E, Little JP. Reduction of AMPK activity and altered MAPKs signalling in peripheral blood mononuclear cells in response to acute glucose ingestion following a short-term high fat diet in young healthy men. Metabolism: clinical and experimental. 2014; 63(9):1209-16. Epub 2014/07/20. 10.1016/j.metabol.2014.06.007 [PubMed: 25037151]

15. Song HJ, Cho YG, Lee HJ. Dietary sodium intake and prevalence of overweight in adults. Metabolism: clinical and experimental. 2013; 62(5):703-8. Epub 2013/01/30. 10.1016/j.metabol. 2012.11.009 [PubMed: 23357528]

16. Kontogianni MD, Vlassopoulos A, Gatzieva A, Farmaki AE, Katsiougiannis S, Panagiotakos DB, et al. Flaxseed oil does not affect inflammatory markers and lipid profile compared to olive oil, in young, healthy, normal weight adults. Metabolism: clinical and experimental. 2013; 62(5):686-93. Epub 2013/01/26. 10.1016/j.metabol.2012.11.007 [PubMed: 23347535]

17. Neale EP, Muhlhausler B, Probst YC, Batterham MJ, Fernandez F, Tapsell LC. Short-term effects of fish and fish oil consumption on total and high molecular weight adiponectin levels in overweight and obese adults. Metabolism: clinical and experimental. 2013; 62(5):651-60. Epub 2012/11/30. 10.1016/j.metabol.2012.10.014 [PubMed: 23190874]

18. Wang CC, Adochio RL, Leitner JW, Abeyta IM, Draznin B, Cornier MA. Acute effects of different diet compositions on skeletal muscle insulin signalling in obese individuals during caloric restriction. Metabolism: clinical and experimental. 2013; 62(4):595-603. Epub 2012/11/24. 10.1016/j.metabol.2012.10.010 [PubMed: 23174405]

19. Rinnankoski-Tuikka R, Hulmi JJ, Torvinen S, Silvennoinen M, Lehti M, Kivela R, et al. Lipid droplet-associated proteins in high-fat fed mice with the effects of voluntary running and diet change. Metabolism: clinical and experimental. 2014; 63(8):1031-40. Epub 2014/06/29. 10.1016/ j.metabol.2014.05.010 [PubMed: 24972504]

20. Wang N, Luo LL, Xu JJ, Xu MY, Zhang XM, Zhou XL, et al. Obesity accelerates ovarian follicle development and follicle loss in rats. Metabolism: clinical and experimental. 2014; 63(1):94-103. Epub 2013/10/19. 10.1016/j.metabol.2013.09.001 [PubMed: 24135502] 
21. Sacre JW, Jellis CL, Jenkins C, Haluska BA, Baumert M, Coombes JS, et al. A six-month exercise intervention in subclinical diabetic heart disease: Effects on exercise capacity, autonomic and myocardial function. Metabolism: clinical and experimental. 2014; 63(9):1104-14. Epub 2014/07/07. 10.1016/j.metabol.2014.05.007 [PubMed: 24997499]

22. Huh JY, Mougios V, Skraparlis A, Kabasakalis A, Mantzoros CS. Irisin in response to acute and chronic whole-body vibration exercise in humans. Metabolism: clinical and experimental. 2014; 63(7):918-21. Epub 2014/05/13. 10.1016/j.metabol.2014.04.001 [PubMed: 24814685]

23. Bluher S, Petroff D, Wagner A, Warich K, Gausche R, Klemm T, et al. The one year exercise and lifestyle intervention program KLAKS: Effects on anthropometric parameters, cardiometabolic risk factors and glycemic control in childhood obesity. Metabolism: clinical and experimental. 2014; 63(3):422-30. Epub 2014/01/11. 10.1016/j.metabol.2013.11.016 [PubMed: 24405751]

24. Saunders TJ, Chaput JP, Goldfield GS, Colley RC, Kenny GP, Doucet E, et al. Prolonged sitting and markers of cardiometabolic disease risk in children and youth: a randomized crossover study. Metabolism: clinical and experimental. 2013; 62(10):1423-8. Epub 2013/06/19. 10.1016/ j.metabol.2013.05.010 [PubMed: 23773981]

25. Numao S, Kawano H, Endo N, Yamada Y, Konishi M, Takahashi M, et al. Effects of a single bout of aerobic exercise on short-term low-carbohydrate/high-fat intake-induced postprandial glucose metabolism during an oral glucose tolerance test. Metabolism: clinical and experimental. 2013; 62(10):1406-15. Epub 2013/06/15. 10.1016/j.metabol.2013.05.005 [PubMed: 23764436]

26. Moon HS, Dincer F, Mantzoros CS. Pharmacological concentrations of irisin increase cell proliferation without influencing markers of neurite outgrowth and synaptogenesis in mouse H197 hippocampal cell lines. Metabolism: clinical and experimental. 2013; 62(8):1131-6. Epub 2013/05/15. 10.1016/j.metabol.2013.04.007 [PubMed: 23664146]

27. Fain JN, Company JM, Booth FW, Laughlin MH, Padilla J, Jenkins NT, et al. Exercise training does not increase muscle FNDC5 protein or mRNA expression in pigs. Metabolism: clinical and experimental. 2013; 62(10):1503-11. Epub 2013/07/09. 10.1016/j.metabol.2013.05.021 [PubMed: 23831442]

28. Tremblay AJ, Lamarche B, Deacon CF, Weisnagel SJ, Couture P. Effects of sitagliptin therapy on markers of low-grade inflammation and cell adhesion molecules in patients with type 2 diabetes. Metabolism: clinical and experimental. 2014; 63(9):1141-8. Epub 2014/07/19. 10.1016/j.metabol. 2014.06.004 [PubMed: 25034387]

29. Garrido P, Salehzadeh F, Duque-Guimaraes DE, Al-Khalili L. Negative regulation of glucose metabolism in human myotubes by supraphysiological doses of 17 beta-estradiol or testosterone. Metabolism: clinical and experimental. 2014; 63(9):1178-87. Epub 2014/07/19. 10.1016/ j.metabol.2014.06.003 [PubMed: 25034385]

30. Simar D, Versteyhe S, Donkin I, Liu J, Hesson L, Nylander V, et al. DNA methylation is altered in $\mathrm{B}$ and NK lymphocytes in obese and type 2 diabetic human. Metabolism: clinical and experimental. 2014; 63(9):1188-97. Epub 2014/07/06. 10.1016/j.metabol.2014.05.014 [PubMed: 24996265]

31. del Rosario MC, Ossowski V, Knowler WC, Bogardus C, Baier LJ, Hanson RL. Potential epigenetic dysregulation of genes associated with MODY and type 2 diabetes in humans exposed to a diabetic intrauterine environment: an analysis of genome-wide DNA methylation. Metabolism: clinical and experimental. 2014; 63(5):654-60. Epub 2014/03/04. 10.1016/j.metabol. 2014.01.007 [PubMed: 24582139]

32. Choe EY, Wang HJ, Kwon O, Kim KJ, Kim BS, Lee BW, et al. Variants of the adiponectin gene and diabetic microvascular complications in patients with type 2 diabetes. Metabolism: clinical and experimental. 2013; 62(5):677-85. Epub 2012/12/25. 10.1016/j.metabol.2012.11.005 [PubMed: 23260797]

33. Geloneze B, da de Oliveira MS, Vasques AC, Novaes FS, Pareja JC, Tambascia MA. Impaired incretin secretion and pancreatic dysfunction with older age and diabetes. Metabolism: clinical and experimental. 2014; 63(7):922-9. Epub 2014/05/24. 10.1016/j.metabol.2014.04.004 [PubMed: 24854384]

34. Pratchayasakul W, Chattipakorn N, Chattipakorn SC. Estrogen restores brain insulin sensitivity in ovariectomized non-obese rats, but not in ovariectomized obese rats. Metabolism: clinical and 
experimental. 2014; 63(6):851-9. Epub 2014/04/20. 10.1016/j.metabol.2014.03.009 [PubMed: 24742706]

35. van Beek L, Lips MA, Visser A, Pijl H, Ioan-Facsinay A, Toes R, et al. Increased systemic and adipose tissue inflammation differentiates obese women with T2DM from obese women with normal glucose tolerance. Metabolism: clinical and experimental. 2014; 63(4):492-501. Epub 2014/01/29. 10.1016/j.metabol.2013.12.002 [PubMed: 24467914]

36. Liu A, Kushida CA, Reaven GM. Habitual shortened sleep and insulin resistance: an independent relationship in obese individuals. Metabolism: clinical and experimental. 2013; 62(11):1553-6. Epub 2013/07/16. 10.1016/j.metabol.2013.06.003 [PubMed: 23849514]

37. Erdogan D, Yucel H, Uysal BA, Ersoy IH, Icli A, Akcay S, et al. Effects of prediabetes and diabetes on left ventricular and coronary microvascular functions. Metabolism: clinical and experimental. 2013; 62(8):1123-30. Epub 2013/04/06. 10.1016/j.metabol.2013.02.011 [PubMed: 23557591]

38. Panagiotou G, Mu L, Na B, Mukamal KJ, Mantzoros CS. Circulating irisin, omentin-1, and lipoprotein subparticles in adults at higher cardiovascular risk. Metabolism: clinical and experimental. 2014 Epub 2014/07/26. 10.1016/j.metabol.2014.06.001

39. Kuwashiro T, Ago T, Kamouchi M, Matsuo R, Hata J, Kuroda J, et al. Significance of plasma adiponectin for diagnosis, neurological severity and functional outcome in ischemic stroke Research for Biomarkers in Ischemic Stroke (REBIOS). Metabolism: clinical and experimental. 2014; 63(9):1093-103. Epub 2014/06/16. 10.1016/j.metabol.2014.04.012 [PubMed: 24929894]

40. Nakatsuji H, Kishida K, Sekimoto R, Komura N, Kihara S, Funahashi T, et al. Accumulation of adiponectin in inflamed adipose tissues of obese mice. Metabolism: clinical and experimental. 2014; 63(4):542-53. Epub 2014/01/29. 10.1016/j.metabol.2013.12.012 [PubMed: 24467915]

41. Aprahamian TR. Elevated adiponectin expression promotes adipose tissue vascularity under conditions of diet-induced obesity. Metabolism: clinical and experimental. 2013; 62(12):1730-8. Epub 2013/09/03. 10.1016/j.metabol.2013.07.010 [PubMed: 23993424]

42. Liu X, Hamnvik OP, Chamberland JP, Petrou M, Gong H, Christophi CA, et al. Circulating alanine transaminase (ALT) and gamma-glutamyl transferase (GGT), but not fetuin-A, are associated with metabolic risk factors, at baseline and at two-year follow-up: the prospective Cyprus Metabolism Study. Metabolism: clinical and experimental. 2014; 63(6):773-82. Epub 2014/04/15. 10.1016/ j.metabol.2014.03.008 [PubMed: 24726813]

43. Polyzos SA, Kountouras J, Anastasilakis AD, Geladari EV, Mantzoros CS. Irisin in patients with nonalcoholic fatty liver disease. Metabolism: clinical and experimental. 2014; 63(2):207-17. Epub 2013/10/22. 10.1016/j.metabol.2013.09.013 [PubMed: 24140091]

44. Crujeiras AB, Zulet MA, Lopez-Legarrea P, de la Iglesia R, Pardo M, Carreira MC, et al. Association between circulating irisin levels and the promotion of insulin resistance during the weight maintenance period after a dietary weight-lowering program in obese patients. Metabolism: clinical and experimental. 2014; 63(4):520-31. Epub 2014/01/21. 10.1016/j.metabol.2013.12.007 [PubMed: 24439241]

45. Swick AG, Orena S, O'Connor A. Irisin levels correlate with energy expenditure in a subgroup of humans with energy expenditure greater than predicted by fat free mass. Metabolism: clinical and experimental. 2013; 62(8):1070-3. Epub 2013/04/13. 10.1016/j.metabol.2013.02.012 [PubMed: 23578923]

46. Hwang JJ, Thakkar B, Chamberland JP, Mantzoros CS. Circulating fetuin-A levels are not affected by short and long-term energy deprivation and/or by leptin administration. Metabolism: clinical and experimental. 2014; 63(6):754-9. Epub 2014/04/08. 10.1016/j.metabol.2014.02.006 [PubMed: 24703486]

47. Holmstrom MH, Tom RZ, Bjornholm M, Garcia-Roves PM, Zierath JR. Effect of leptin treatment on mitochondrial function in obese leptin-deficient ob/ob mice. Metabolism: clinical and experimental. 2013; 62(9):1258-67. Epub 2013/05/15. 10.1016/j.metabol.2013.04.001 [PubMed: 23664724]

48. Cassano S, Pucino V, La Rocca C, Procaccini C, De Rosa V, Marone G, et al. Leptin modulates autophagy in human CD4CD25 conventional T cells. Metabolism: clinical and experimental. 2014 Epub 2014/07/26. 10.1016/j.metabol.2014.06.010 
49. Dalamaga M, Karmaniolas K, Chamberland J, Nikolaidou A, Lekka A, Dionyssiou-Asteriou A, et al. Higher fetuin-A, lower adiponectin and free leptin levels mediate effects of excess body weight on insulin resistance and risk for myelodysplastic syndrome. Metabolism: clinical and experimental. 2013; 62(12):1830-9. Epub 2013/10/22. 10.1016/j.metabol.2013.09.007 [PubMed: 24140093]

50. Aronis KN, Sahin-Efe A, Chamberland JP, Spiro A 3rd, Vokonas P, Mantzoros CS. Chemerin levels as predictor of acute coronary events: a case-control study nested within the veterans affairs normative aging study. Metabolism: clinical and experimental. 2014; 63(6):760-6. Epub 2014/04/02. 10.1016/j.metabol.2014.02.013 [PubMed: 24684821]

51. Hondares E, Gallego-Escuredo JM, Flachs P, Frontini A, Cereijo R, Goday A, et al. Fibroblast growth factor-21 is expressed in neonatal and pheochromocytoma-induced adult human brown adipose tissue. Metabolism: clinical and experimental. 2014; 63(3):312-7. Epub 2013/12/29. 10.1016/j.metabol.2013.11.014 [PubMed: 24369918]

52. Oberbach A, Neuhaus J, Inge T, Kirsch K, Schlichting N, Bluher S, et al. Bariatric surgery in severely obese adolescents improves major comorbidities including hyperuricemia. Metabolism: clinical and experimental. 2014; 63(2):242-9. Epub 2013/12/18. 10.1016/j.metabol.2013.11.012 [PubMed: 24332707]

53. Davis CR, Dearing E, Usher N, Trifiletti S, Zaichenko L, Ollen E, et al. Detailed assessments of childhood adversity enhance prediction of central obesity independent of gender, race, adult psychosocial risk and health behaviors. Metabolism: clinical and experimental. 2014; 63(2):199206. Epub 2013/11/12. 10.1016/j.metabol.2013.08.013 [PubMed: 24211017]

54. Krolow R, Noschang C, Arcego DM, Huffell AP, Marcolin ML, Benitz AN, et al. Sex-specific effects of isolation stress and consumption of palatable diet during the prepubertal period on metabolic parameters. Metabolism: clinical and experimental. 2013; 62(9):1268-78. Epub 2013/05/15. 10.1016/j.metabol.2013.04.009 [PubMed: 23664084]

55. Glueck CJ, Morrison JA, Wang P, Woo JG. Early and late menarche are associated with oligomenorrhea and predict metabolic syndrome 26 years later. Metabolism: clinical and experimental. 2013; 62(11):1597-606. Epub 2013/08/21. 10.1016/j.metabol.2013.07.005 [PubMed: 23953892]

56. Malloy VL, Perrone CE, Mattocks DA, Ables GP, Caliendo NS, Orentreich DS, et al. Methionine restriction prevents the progression of hepatic steatosis in leptin-deficient obese mice. Metabolism: clinical and experimental. 2013; 62(11):1651-61. Epub 2013/08/10. 10.1016/j.metabol. 2013.06.012 [PubMed: 23928105]

57. Aronis KN, Chamberland JP, Mantzoros CS. GLP-1 promotes angiogenesis in human endothelial cells in a dose-dependent manner, through the Akt, Src and PKC pathways. Metabolism: clinical and experimental. 2013; 62(9):1279-86. Epub 2013/05/21. 10.1016/j.metabol.2013.04.010 [PubMed: 23684008]

58. Holstila M, Virtanen KA, Gronroos TJ, Laine J, Lepomaki V, Saunavaara J, et al. Measurement of brown adipose tissue mass using a novel dual-echo magnetic resonance imaging approach: a validation study. Metabolism: clinical and experimental. 2013; 62(8):1189-98. Epub 2013/04/17. 10.1016/j.metabol.2013.03.002 [PubMed: 23587549]

59. d'Assignies G, Fontes G, Kauffmann C, Latour M, Gaboury L, Boulanger Y, et al. Early detection of liver steatosis by magnetic resonance imaging in rats infused with glucose and intralipid solutions and correlation to insulin levels. Metabolism: clinical and experimental. 2013; 62(12): 1850-7. Epub 2013/09/17. 10.1016/j.metabol.2013.08.003 [PubMed: 24035445]

60. Schopman JE, Admiraal WM, Soeters MR, Ackermans MT, Bisschop PL, Frier BM, et al. Ffluorodeoxyglucose uptake in brown adipose tissue during insulin-induced hypoglycemia and mild cold exposure in non-diabetic adults. Metabolism: clinical and experimental. 2014 Epub 2014/08/15. 10.1016/j.metabol.2014.06.017 